\title{
Travelling Waves in Space-Fractional Nonlinear Diffusion with Linear Convection
}

\author{
Felix Silva Costa1, Marta Raquel Araujo Pereira² \\ ${ }^{1}$ Department of Mathematics and Informatics, State University of Maranhão, São Luis, Brazil \\ ${ }^{2}$ Computer Engineering, State University of Maranhão, São Luis, Brazil \\ Email: felix@cecen.uema.br, marta.raquel@engecomp.uema.br
}

How to cite this paper: Costa, F.S. and Pereira, M.R.A. (2017) Travelling Waves in Space-Fractional Nonlinear Diffusion with Linear Convection. Journal of Applied Mathematics and Physics, 5, 462-468. https://doi.org/10.4236/jamp.2017.52041

Received: December 30, 2016

Accepted: February 21, 2017

Published: February 24, 2017

Copyright $\odot 2017$ by authors and Scientific Research Publishing Inc. This work is licensed under the Creative Commons Attribution International License (CC BY 4.0).

http://creativecommons.org/licenses/by/4.0/

c) (i) Open Access

\begin{abstract}
In this paper we investigate anomalous diffusion coupled with linear convection, using fractional calculus to describe the anomalous associated memory effects in diffusive term. We get an explicit travelling wave solution, wavefront, with finite propagation. We comment the properties of the solution, including the stationary case.
\end{abstract}

\section{Keywords}

Fractional Convection-Diffusion, Riesz Derivative, Travelling Wave, Wavefront

\section{Introduction}

The fractional calculus is almost as old as the calculus of integer order, but only in 1974 was realized the first Conference on Fractional Calculus its Applications. In the decade 90s fractional calculus was recognized and specific journals and textbooks were being published [1] [2] [3]. Nowadays, many definitions have appeared in fractional calculus that shows us some difficulties and limitations of the theory with applications [4] [5] [6] [7]. The researchers have investigated in diffusion processes the anomalous related memory effects [8] [9] [10] [11], for example, some materials the moisture propagates according to the $x / t^{\alpha / 2}$ scaling, with $0<\alpha<2$ [12] [13]. Costa et al. [14] comment that water transport for large distance in a relatively short time (groundwater infiltration problem) can be described for a fractional space-time nonlinear diffusion equation.

We study the diffusion with linear convection, that is, a nonlinear convection-diffusion problem. Appearing in several physical, biological and chemical applications, B. H. Gilding and R. Kersner [15] highlight the study of pattern formation by bacterial colonies analyzed in [16]. The investigation of that problem with the fractional operators has shown that fractional derivatives in con- 
vection-diffusion equation give more information about the anomalous associated with effect memory [17] [18] [19]. We are interested in travelling wave solution given by similarity reductions to fractional equation.

Our purpose is to find an explicit solution, so that we can investigate its properties in the fractional model. In Section 2 we define the Riesz fractional derivative. In Section 3 the fractional model used to describe the convection-diffusion equation using Riesz fractional derivative. In Section 4 we use the similarity reduction method in fractional equation. In Section 5 we calculate the particular travelling wave, called a wavefront. Section 6 makes our concluding remarks.

\section{Riesz Fractional Derivative}

The Riesz fractional derivative of order $\alpha$, with $0<\alpha<2$ and $\alpha \neq 1$ is defined by:

$$
D_{x}^{\alpha} f(x)=-\frac{D_{+}^{\alpha} f(x)+D_{-}^{\alpha} f(x)}{2 \cos (\alpha \pi / 2)},
$$

where $D_{ \pm}^{\alpha} f(x)$ are Weyl fractional derivatives [20] [21].

Theorem 1. Let be $h(x)=|x|^{-\alpha-1}$, with $1<\alpha<2$. We describe the Riesz fractional derivative of order $\alpha$ for an appropriate Fourier convolution product is given by.

$$
D_{x}^{\alpha} f(x)=d_{\alpha}(f * h)(x),
$$

with $d_{\alpha}=-\frac{1}{2 \Gamma(-\alpha) \cos \left(\frac{\alpha \pi}{2}\right)}$.

This result is an improvement of the theorem developed by E. C. Grigoletto and E. C. de Oliveira [22].

\section{Fractional Convection-Diffusion}

Analyze a fractional space nonlinear convection-diffusion equation, in which we apply the Riesz fractional derivative in diffusive term. If we consider only diffusion, Equation (3) defines a fractional porous medium equation [23] [24] [25].

$$
\frac{\partial}{\partial t} u(x, t)=D_{x}^{\alpha} u^{n}(x, t)+\frac{\partial}{\partial x} u(x, t), n>1, t>0 \text { and } x \in \mathbb{R},
$$

where $D_{x}^{\alpha}$ is Riesz fractional derivative, with $1<\alpha<2$. In the case $\alpha \rightarrow 2$, we get the integer classical convection-diffusion equation. The parameter $n$ appears, when we consider the diffusion coefficient $n u^{n-1}(x, t)$, that is, density dependent diffusivity, in that $n$ is determined by properties of the medium and phenomenon investigated.

An important application arises in a model describing the unsaturated flow of a fluid through a homogeneous porous column under the influence of capillary pressure and gravity. As example, we cite water movement in a vertical column of the medium, if water movement in a horizontal column of the medium, the problem reduces to the flow porous medium. 


\section{Travelling waves}

Use the similarity reduction method in Equation (3), to find the travelling wave solutions $g(x \pm c t)$, where $g(x \pm c t)=x^{d} g\left(1 \pm c \frac{t}{x}\right)$, with $d$ is related to the homogeneity degree.

$$
u(x, t)=x^{a} U(\eta), \quad \eta=1 \pm c \frac{t}{x},
$$

where $t>0, x \in \mathbb{R}-\{0\}$ and $a$ is unknown.

We apply Equation (4) on the left-side hand of Equation (3):

$$
\frac{\partial}{\partial t} u(x, t)=\frac{\partial}{\partial t}\left[x^{a} U\left(1 \pm c \frac{t}{x}\right)\right]=( \pm c) x^{a-1} \frac{\partial}{\partial \eta} U(\eta) .
$$

In relation to the right-side hand of Equation (3). We calculate Riesz fractional derivative, using Theorem 1:

$$
\begin{aligned}
D_{x}^{\alpha} u^{n}(x, t) & =D_{x}^{\alpha}\left[x^{a n} U^{n}\left(1 \pm c \frac{t}{x}\right)\right]=d_{\alpha}\left[x^{a n} U^{n}\left(1 \pm c \frac{t}{x}\right)\right] *|x|^{-\alpha-1} \\
& =d_{\alpha} \int_{-\infty}^{+\infty} s^{a n} U^{n}\left(1 \pm c \frac{t}{s}\right)|x-s|^{-\alpha-1} \mathrm{~d} s \\
& =d_{\alpha}\left[\int_{x}^{+\infty}(s-x)^{-\alpha-1} s^{a n} U^{n}\left(1 \pm c \frac{t}{s}\right) \mathrm{d} s+\int_{-\infty}^{x}(x-s)^{-\alpha-1} s^{a n} U^{n}\left(1 \pm c \frac{t}{s}\right) \mathrm{d} s\right]
\end{aligned}
$$

The integrals are given follows:

$$
\begin{aligned}
& \int_{-\infty}^{x}(x-s)^{-\alpha-1} s^{a n} U^{n}\left(1 \pm c \frac{t}{s}\right) \mathrm{d} s \\
& =x^{a n-\alpha}(1-\eta)^{1+a n} \int_{1}^{\eta}(\eta-\tau)^{-1-\alpha}(1-\tau)^{-1+\alpha-a n} U^{n}(\tau) \mathrm{d} \tau
\end{aligned}
$$

and

$$
\begin{aligned}
& \int_{x}^{+\infty}(s-x)^{-\alpha-1} s^{a n} U^{n}\left(1 \pm c \frac{t}{s}\right) \mathrm{d} s \\
& =(-1)^{-\alpha} x^{a n-\alpha}(1-\eta)^{1+a n} \int_{1}^{\eta}(\eta-\psi)^{-1-\alpha}(1-\psi)^{-1+\alpha-a n} U^{n}(\psi) \mathrm{d} \psi
\end{aligned}
$$

The Riesz fractional derivative is given by:

$$
\begin{aligned}
& D_{x}^{\alpha} u^{n}(x, t) \\
& =d_{\alpha}\left[1+(-1)^{-\alpha}\right] x^{a n-\alpha}(1-\eta)^{1+a n} \int_{1}^{\eta}(\eta-\psi)^{-1-\alpha}(1-\psi)^{-1+\alpha-a n} U^{n}(\psi) \mathrm{d} \psi .
\end{aligned}
$$

Replacing, Equation (5) and Equation (9) in Equation (3), we get:

$$
\begin{aligned}
& ( \pm c) x^{a-1} \frac{\partial}{\partial \eta} U(\eta) \\
& =d_{\alpha}\left[1+(-1)^{-\alpha}\right] x^{a n-\alpha}(1-\eta)^{1+a n} \int_{1}^{\eta}(\eta-\psi)^{-1-\alpha}(1-\psi)^{-1+\alpha-a n} U^{n}(\psi) \mathrm{d} \psi \\
& \quad+x^{a-1}[a+(1-\eta)] \frac{\partial}{\partial \eta} U(\eta)
\end{aligned}
$$

Imposing invariance on the variable $x$ for Equation (10): 


$$
a-1=a n-\alpha \Rightarrow a=\frac{\alpha-1}{n-1}
$$

We obtain the equation:

$$
\begin{aligned}
& ( \pm c) \frac{\partial}{\partial \eta} U(\eta) \\
& =-d_{\alpha}\left[1+(-1)^{-\alpha}\right](1-\eta)^{1+a n} \int_{\eta}^{1}(\eta-\psi)^{-1-\alpha}(1-\psi)^{-1+\alpha-a n} U^{n}(\psi) \mathrm{d} \psi \\
& +\left[a+(1-\eta) \frac{\partial}{\partial \eta}\right] U(\eta)
\end{aligned}
$$

\section{Wavefronts}

Look for a travelling wave, called wavefront [26] [27] [28]. Let us consider:

$$
U(\eta)= \begin{cases}A \eta^{k}, & \eta<0 \\ 0, & \eta \geq 0\end{cases}
$$

where $A$ and $k$ are unknowns. To determine these variables, we replace this equation in Equation (12). The calculus of the integral is given by:

$$
\begin{aligned}
& d_{\alpha}\left[1+(-1)^{-\alpha}\right](1-\eta)^{1+a n} \int_{\eta}^{1}(\eta-\psi)^{-1-\alpha}(1-\psi)^{-1+\alpha-a n} U^{n}(\psi) \mathrm{d} \psi= \\
= & d_{\alpha}\left[1+(-1)^{-\alpha}\right](1-\eta)^{1+a n} \int_{\eta}^{0}(\eta-\psi)^{-1-\alpha}(1-\psi)^{-1+\alpha-a n} A^{n} \psi^{\kappa n} \mathrm{~d} \psi \\
= & d_{\alpha}\left[1+(-1)^{-\alpha}\right] \eta^{\kappa n-\alpha}(1-\eta)^{1+a n} A^{n} \int_{1}^{0}(1-\mu)^{-1-\alpha}(1-\eta \mu)^{-1+\alpha-a n} \mu^{\kappa n} \mathrm{~d} \mu \\
= & d_{\alpha}\left[1+(-1)^{-\alpha}\right] A^{n} \eta^{k n-\alpha} \\
& \times(1-\eta)^{1+a n} \frac{(-1) \Gamma(-\alpha) \Gamma(1+\kappa n)}{\Gamma(1-\alpha+\kappa n)} F_{1}(1-\alpha+a n, 1+\kappa n ; 1-\alpha+\kappa n ; \eta),
\end{aligned}
$$

where ${ }_{2} F_{1}$ is hypergeometric function. Thus, we obtain the relation:

$$
\begin{aligned}
& ( \pm c) A k \eta^{k-1} \\
& =-d_{\alpha}\left[1+(-1)^{-\alpha}\right] A^{n} \eta^{k n-\alpha} \\
& \quad \times(1-\eta)^{1+a n} \frac{(-1) \Gamma(-\alpha) \Gamma(1+k n)}{\Gamma(1-\alpha+k n)} F_{1}(1-\alpha+a n, 1+k n ; 1-\alpha+k n ; \eta) \\
& +A\left[(a-k) \eta^{k}+k \eta^{k-1}\right] .
\end{aligned}
$$

Once again, considering $k=a$, we impose invariance on the variable $\eta$ for Equation (15), that gives us:

$$
( \pm c) k A=-d_{\alpha}\left[1+(-1)^{-\alpha}\right] A^{n} \frac{(-1) \Gamma(-\alpha) \Gamma(1+k n)}{\Gamma(1-\alpha+k n)}+k A .
$$

Thus,

$$
A^{n-1}=-\frac{\exp (i \alpha \pi / 2) \Gamma(1-\alpha+k n)}{\Gamma(1+k n)} k[( \pm c)-1]
$$

and using Equation (11), we have: 


$$
A=\left\{-\frac{\exp (i \alpha \pi / 2) \Gamma(1+k)}{\Gamma(1+k n)}[( \pm c)-1]\right\}^{\frac{1}{n-1}} .
$$

The values of $A$ and $a$ in Equation (4),

$$
u(x, t)= \begin{cases}\left\{-\frac{\exp (i \alpha \pi / 2) \Gamma(1+k)}{\Gamma(1+k n)}[( \pm c)-1](x \pm c t)^{\alpha-1}\right\}^{\frac{1}{n-1}}, & |x|<c t \\ 0, & |x| \geq c t .\end{cases}
$$

The integer classical equation is recovered by taking the limit $\alpha \rightarrow 2$ in Equation (3):

$$
\frac{\partial}{\partial t} u(x, t)=\frac{\partial^{2}}{\partial x^{2}} u^{n}(x, t)+\frac{\partial}{\partial x} u(x, t),
$$

and its solution is given by:

$$
u(x, t)= \begin{cases}\left\{\frac{(n-1)}{n}[( \pm c)-1](x \pm c t)\right\}^{\frac{1}{n-1}}, & |x|<c t \\ 0, & |x| \geq c t,\end{cases}
$$

which is same result found by B.H. Gilding and R. Kersner in [27].

The condition $\eta<0$ gives us the relations: $x \in(0, \infty)$ associated to " $-c$ " and $x \in(-\infty, 0)$ associated to " $+c$ ", that is, $x \rightarrow-x$ implies the change of velocity $-c \rightarrow+c$. If we regard $u(x, t)$ in Equation (21) nonnegative, we have $c \geq 0 x \in(0, \infty)$ and $c>1 x \in(-\infty, 0)$. Therefore, the fractional equation in Equation (3) has only stationary solution in $(0, \infty)$. The relation between the values $A$ and $c$ is establishes in Equation (18).

\section{Concluding Remarks}

We introduce the space-fractional model based on Riesz fractional derivative with nonlinear diffusion in conjunction linear convection. We obtain the explicit travelling wave solution, with finite propagation, wavefront; that is, Equation (3) admits solution in the region $x<c t$ such that $u(x, t)$ vanishes on the front $x=c t$. This property and the behaviour near the interface are in general described by wavefront; it may give rise to interfaces separating the regions that we have just mentioned. As a continuation of this paper, we can suppose fractional derivatives in time and nonlinear convection for Equation (3) and we search its travelling waves.

\section{Acknowledgements}

The authors are grateful to the referee and Dr. A. J. Silva for their useful suggestions and discussions.

\section{References}

[1] Machado, J.A.T., Kiryakova, V. and Mainardi, F. (2010) A Poster about the Recent History of Fractional Calculus. Fractional Calculus Applied \& Analysis, 13, 329-334. 
[2] Machado, J.A.T., Kiryakova, V. and Mainardi, F. (2010) A Poster about the Old History of Fractional Calculus. Fractional Calculus \& Applied Analysis, 13, 447-454.

[3] Machado, J.A.T., Kiryakova, V. and Mainardi, F. (2011) Recent History of Fractional Calculus. Communications in Nonlinear Science and Numerical Simulation, 16, 1140-1153. https://doi.org/10.1016/j.cnsns.2010.05.027

[4] Capelas de Oliveira, E. and Machado, J.A.T. (2014) A Review of Definitions For Fractional Derivatives and Integral. Mathematical Problems in Engineering, 2014, 238459. https://doi.org/10.1155/2014/238459

[5] Machado, J.A.T., Mainardi, F. and Kiryakova, V. (2015) Fractional Calculus: Quo vadimus? (Where Are We Going?). Fractional Calculus and Applied Analysis, 18, 495-526. https://doi.org/10.1515/fca-2015-0031

[6] Ortigueira, M.D. and Machado, J.A.T. (2015) What Is a Fractional Derivative? Journal of Computational Physics, 293, 4-13. https://doi.org/10.1016/j.jcp.2014.07.019

[7] Sabatier, J., Merveillaut, M., Malti, R. and Oustaloup, A. (2010) How to Impose Physically Coherent Initial Conditions to a Fractional System? Communications in Nonlinear Science and Numerical Simulation, 15, 1318-1326. https://doi.org/10.1016/j.cnsns.2009.05.070

[8] Costa, F.S. and Capelas de Oliveira, E. (2012) Fractional Wave-Diffusion Equation with Periodic Conditions. Journal of Mathematical Physics, 53, 123520. https://doi.org/10.1063/1.4769270

[9] Costa, F.S., Grigoleto, E.C. and Capelas de Oliveira, E. (2015) Slowing-Down of Neutrons: A Fractional Model. Communications in Applied and Industrial Mathematics, 6, e-538.

[10] Jafari, H. and Momani, S. (2007) Solving Fractional Diffusion and wave Equations by Modified Homotopy Perturbation Method. Physics Letters A, 370, 388-396. https://doi.org/10.1016/j.physleta.2007.05.118

[11] Mehdi Safari, M. and Danesh, M. (2011) Application of Adomian's Decomposition Method for the Analytical Solution of Space Fractional Diffusion Equation. Advances in Pure Mathematics, 1, 345-350. https://doi.org/10.4236/apm.2011.16062

[12] Luncho, Y., Mainardi, F. and Povstenko, Y. (2013) Propagation Speed of the Maximum of the Fundamental Solution to the Fractional Diffusion-Wave Equation. Computers and Mathematics with Applications, 66, 774-784.

https://doi.org/10.1016/j.camwa.2013.01.005

[13] Plociniczak, L. (2015) Analytical Studies of a Time-Fractional Porous Medium Equation. Derivation, Approximation and Applications. Communications in Nonlinear Science and Numerical Simulation, 24, 169-183. https://doi.org/10.1016/j.cnsns.2015.01.005

[14] Costa, F.S., Marão, J.A.P.F., Alves Soares, J.C. and Capelas de Oliveira, E. (2015) Similarity Solution to Fractional Nonlinear Space-Time Diffusion-Wave Equation. Journal of Mathematical Physics, 56, Article ID: 033507.

https://doi.org/10.1063/1.4915293

[15] Gilding, B.H. and Kesner, R. (2005) A Fisher/Kpp-Type Equation with Density-Dependent Diffusion and Convection: Travelling-Wave Solutions. Journal of Physics A: Mathematical and General, 38, 3367-3379.

https://doi.org/10.1088/0305-4470/38/15/009

[16] Cohen, I. Golding, I., Kozlovsky, Y. and Ron, I.G. (1999) Continuous and Discrete Models of Cooperation in Complex Bacterial Colonies. Fractals, 7, 235-247.

[17] Cuesta, C.M. (2015) A Non-Local Kdv-Burgers Equation: Numerical Study of Tra- 
velling Waves. Communications in Applied and Industrial Mathematics, 6, e-533.

[18] Chen, M. and Deng, W. (2014) A Second-Order Numerical Method for Two-Dimensional Two-Sided Space Fractional Convection Diffusion Equation. Applied Mathematical Modelling, 38, 3244-3259. https://doi.org/10.1016/j.apm.2013.11.043

[19] Zhang, J. and Zhang, J. (2015) Symmetries Analysis for Time-Fractional Convection-Diffusion Equation. arXiv preprint arXiv:1512.01319.

[20] Camargo, R.F. and Capelas de Oliveira, E. (2015) Cálculo fracionário. Livraria da Física, São Paulo.

[21] Mainardi, F., Luchko, Y. and Pagnini, G. (2001) The Fundamental Solution of the Space-Time Fractional Diffusion Equation Fractional Calculus Applied and Analysis, 4, 153-192.

[22] Grigoletto, E.C. and Capelas de Oliveira, E. (2013) Fractional Versions of the Fundamental Theorem of Calculus. Applied Mathematics, 4, 23-33.

https://doi.org/10.4236/am.2013.47A006

[23] Biler, P., Imbert, C. and Karch, G. (2015) The Nonlocal Porous Medium Equation: Barenblatt Profiles and Other Weak Solutions. Archive for Rational Mechanics and Analysis, 215, 497-529. https://doi.org/10.1007/s00205-014-0786-1

[24] Pablo, A., Quirós, F., Rodriguíguez, A. and Vázquez, J.L. (2011) A Fractional Porous Medium Equation. Advances in Mathematics, 226, 1378-1409.

https://doi.org/10.1016/j.aim.2010.07.017

[25] Huang, Y. (2014) Explicit Barenblatt Profiles for Fractional Porous Medium Equations. Bulletin London Mathematical Society, 46, 857-869. https://doi.org/10.1112/blms/bdu045

[26] Djordjevic, V.D. and Atanackovic, T.M. (2008) Similarity Solutions to Nonlinear Heat Conduction and Burgers/Korteweg-Devries Fractional Equations. Journal of Computational and Applied Mathematics, 222, 701-714. https://doi.org/10.1016/j.cam.2007.12.013

[27] Gilding, B. and Kersner, R. (2004) Travelling Waves in Nonlinear Diffusion-Convection Reaction. Birkhäuser, Basel. https://doi.org/10.1007/978-3-0348-7964-4

[28] Pablo, A. and Sánchez, A. (2000) Global Travelling Waves in Reaction-ConvectionDiffusion Equations. Journal of Differential Equations, 165, 377-413. https://doi.org/10.1006/jdeq.2000.3781

\section{Submit or recommend next manuscript to SCIRP and we will provide best service for you:}

Accepting pre-submission inquiries through Email, Facebook, LinkedIn, Twitter, etc. A wide selection of journals (inclusive of 9 subjects, more than 200 journals)

Providing 24-hour high-quality service

User-friendly online submission system

Fair and swift peer-review system

Efficient typesetting and proofreading procedure

Display of the result of downloads and visits, as well as the number of cited articles

Maximum dissemination of your research work

Submit your manuscript at: http://papersubmission.scirp.org/

Or contact jamp@scirp.org 